Invited paper accepted for publication in Intelligence

\title{
The Future of Intelligence: The Central Meaning-Making Unit of Intelligence in the Mind, the Brain, and Artificial Intelligence
}

\author{
Andreas Demetriou ${ }^{1,2}$, Hudson Golino ${ }^{3}$, George Spanoudis ${ }^{4}$, \\ Nikolaos Makris ${ }^{5}$, and Samuel Greiff ${ }^{6}$ \\ 1. University of Nicosia, Cyprus
}

2. Cyprus Academy of Sciences, Letters, and Arts, Cyprus.

3. University of Virginia, USA

4. University of Cyprus, Cyprus.

5. Democritus University of Thrace, Greece

6. University of Luxembourg, Luxembourg

Acknowledgement: Thanks are due to Professor Adam Chuderski for providing data for the modeling needs of this paper. Special thanks are due to Professors Jarkko Hautamaki, Michael Shayer, Juan Pascual-Leone, and Robert Siegler and two anonymous reviewers for their constructive comments on an earlier version of this paper.

Correspondence: ademetriou@ucy.ac.cy; Tel.: +35799697199 


\title{
The Future of Intelligence: The Central Meaning-Making Unit of Intelligence in the Mind, the Brain, and Artificial Intelligence
}

\begin{abstract}
This paper focuses on general intelligence, $\mathrm{g}$. We first point to broadly accepted facts about g: it is robust, reliable, and sensitive to learning. We then summarize conflicting theories about its nature and development (Mutualism, Process Overlap Theory, and Dynamic Mental Field Theory) and suggest how future research may resolve their disputes. A model is proposed for g involving a core meaning-making mechanism, noetron, drawing on Alignment, Abstraction, and Cognizance, perpetually generating new mental content. Noetron develops through several levels of control: episodic $\rightarrow$ attentional $\rightarrow$ inferential $\rightarrow$ truth $\rightarrow$ epistemic control in infancy, preschool, childhood, adolescence, and adulthood, respectively. Finally, we propose an agenda for future brain, assuming a brain noetron, and artificial intelligence research, assuming an artificial noetron, that might uncover the underlying brain mechanisms of $g$ and generate artificial general intelligence.
\end{abstract}




\section{The Future of Intelligence: The Central Meaning-Making Unit of Intelligence in the Mind, the Brain, and Artificial Intelligence}

The Editor of this special issue invited authors to foresee the future of intelligence research. To respond, we point to questions, possible answers, and methods of future intelligence research, focusing on general intelligence, g, integrating over psychometric, developmental, and brain research. To set the frame, we first summarize what is accepted about $\mathrm{g}$. Then we highlight issues in dispute, assuming that they will be in the center of future research and outline research for resolving disagreements. Finally, we discuss how artificial intelligence must develop if it will ever approach human intelligence.

For over a century, psychology interpreted intelligence as a function of three types of processes: (i) comprehensors, i.e., the fundamental mechanisms for meaning making, filling gaps in information, and generating new answers, when needed; (ii) enablers, i.e., fundamental processes constraining comprehensors; and (iii) control processes brought to bear on comprehensors and enablers to regulate and improve their operation.

Comprehensors and enablers are with us since Spearman $(1904,1927)$. The eduction of relations and correlates (i.e., induction of relations between entities based on similarities and induction of higher-order relations between relations) is Spearman's comprehensor. This operates under the constraints of mental energy available, i.e., the capacity of the brain to represent and process information. Piaget focused on logical reasoning, explicitly admitting that his stages of cognitive development are, largely, developmental forms of Spearman's eduction mechanism (Piaget, 2001). Therefore, despite differences in language, Piaget's stages are forms of $g$ coming with age. Driven by an asimilation-accomodation mechanism powered by reflective 
abstraction, cognitive development transforms the underlying structure of reversible operations into an increasingly abstract and predictive system able to capture the underlying complexity of the world. This is modeled by structures of set theory and symbolic logic (Piaget, 1970, 2001).

\section{0 ${ }^{\text {th }}$ Century: What We Know about $g$}

A century of research yielded abundant evidence for $\mathrm{g}$. Psychometric $\mathrm{g}$ is a powerful empirical phenomenon, reflecting the positive manifold, the fact that cognitive tests are positively correlated (Spearman, 1904). In the currently dominant Cattell-Horn-Carroll model (CHC), g resides at the apex of a hierarchical structure involving several specific factors, including fluid (gf, inductive and deductive reasoning) and crystallized intelligence (gc, knowledge in different domains), domains of mental efficiency, such as speed, short-term memory, visual processing, auditory processing, problem-solving in different domains, Piagetian reasoning, etc. (Case et al., 2001; Carroll, 1993; Rindermann \& Ackerman, 2020). g predicts important life outcomes, such as school and professional performance, income, etc. (Gottfredson, 2002). Several psychometric tests, such as the WISC or the Raven test and scholastic achievement tests, such as the SAT, are good measures of $\mathrm{g}$ (Jensen, 1998).

Elementary processes reflecting Spearman's mental energy (Jensen, 1998, 2006), such as processing speed, are related with individual differences (Jensen, 2006) and developmental changes in $\mathrm{g}$ (Kail, 2000), accounting for a significant amount of variance in $\mathrm{g}$ (Conway et al., 2002). Also, aspects of attention control, such as inhibition and shifting (Diamond, 2013), and organizational processes in working memory (Baddelley, 2012) are strong indexes of $\mathrm{g}$. Various forms of reasoning reflecting gf are also highly related with g (Gustafsson, 1984). Metacognition and awareness of mental processes are also related with $\mathrm{g}$ (Demetriou \& Kazi, 2006). Thus, the variance of $\mathrm{g}$ can be broken down as an additive function of these processes, which cumulatively 
account for almost $100 \%$ of its variance, ranging from $3 \%$ (speed) to $30 \%$ (working memory) (Chuderki et al., 2014; Demetriou et al., 2018b; Makris et al., 2017).

All processes change through the life span. From infancy to early adulthood, speed increases (Kail, 2000); attention control becomes increasingly flexible and self-directed (Diamond, 2012); working memory expands (Cowan, 2016; Case, 1985; Pascual-Leone \& Johnson, 2021); reasoning becomes increasingly complex and abstract (Moshman, 2011); cognizance becomes increasingly accurate and differentiated (Demetriou et al., 2018a). They then stabilize until early middle age, when they start declining, often at varying onset times and rates (Salthouse, 2016).

Domain specificity and g co-exist. Thus, integration mechanisms in $g$ interact with the precision of representation of critical relations in different domains (Demetriou, Greiff, et al., 2020). In language, reading is difficult for individuals impaired in representing relations between visual patterns and sounds, such as letter-phoneme relations (Dehaene, 2010). In mathematics, grasping arithmetic relations is difficult for individuals impaired in magnitude representation (Butterworth, 2010). In space, grasping spatial relations is difficult for individuals impaired in visualizing spatial relations (Khooshabeh et al., 2013). Social relations may be impaired for individuals with difficulties in representing others' mental states (Hughes \& Leekham, 2004).

Learning may change g. The Flynn (2009) effect reflects the secular improvement of IQ, partly standing for $\mathrm{g}$, by about $15-20$ points over $20^{\text {th }}$ century. Reasoning became more abstract and complex due to enhanced social, occupational, and educational experiences since $19^{\text {th }}$ century. However, at the individual level, intelligence proved very resistant to change. Both large scale and laboratory-based experiments aiming to increase IQ proved unsuccessful. Even when obtained, increases are short-lived, fading out in about 3 years (Protzko, 2015). It is practically 
important to note, however, that $\mathrm{g}$ is highly predictive of school achievement, accounting for about $30 \%$ of variance in school grades (Demetriou, Kazi et al., 2019, 2020), and it changes because of it (Cliffordson \& Gustafsson, 2008).

\section{The Future of Intelligence: What is g?}

The empirical facts above are generally accepted but the nature of $\mathrm{g}$ is strongly disputed for a century. This is reflected in conflicting theories about $\mathrm{g}$. Does $\mathrm{g}$ stand for any specific psychological process or is it just a mathematical index of the state and interactions between different processes? Currently, there are three alternative theories. The standard theory, already summarized, takes inference as the central process, and reduces its operation additively to various enablers. The second, mutualism, recognizes all individual processes but it claims that $g$ per se does not stand for any psychological process; it is an emergent property of interactions between these processes. The third, Dynamic Mental Field Theory (DMFT), stands in between. It claims that $\mathrm{g}$ is a psychological entity including core processes operating together as a meaning-making unit. Basic inference is part of this core, but systematic syllogistic reasoning is not; advanced reasoning in different forms (see Moshman, 2011) comes with development and learning generating overlapping layers of meaning-making tools. The dispute between the three theories will fuel research in the future.

\section{Mutualism}

Mutualism expresses a more general current trend in psychology postulating that general factors abstracted from a variety of measures by factor analytic methods do not represent any psychological process as such. Rather, general factors index the relations between the processes involved, such as psychometric g (Savi et al., 2019; van der Maas et al., 2006, 2017), the general 
factor of personality (Revelle \& Wilt, 2013), or the general factor of psychopathology (van Bork et al., 2017). This line of research uses network modeling to capture the structure of the processes of interest (Epskamp et al., 2018). Networks are topological structures representing the entities of interest (e.g., cognitive process) and their possible links. For instance, Fig. 1A shows the network representing performance on the tasks used by Chuderski et al. (2014) to explore the role of executive processes in $\mathrm{g}$. In this study, each of the following processes was addressed by three tasks: attention control, short-term memory, complex memory span, n-back working memory, relational integration, and fluid intelligence. Each task is represented by one node and their relations are represented by edges (the lines) connecting them. Both nodes and edges are equally important for modeling the processes concerned. Structure emerges from the connections between nodes. For instance, domains, if present, are signified by stronger relations between nodes standing for component processes in each domain (e.g., different forms of working memory) than with nodes standing for processes in other domains (e.g., different forms of reasoning). There is no hierarchical structure or factor in the network. Thus, there is no latent variable separately standing for g; literally speaking, according to proponents of network modeling, g, as such, does not exist in networks. However, some nodes may occupy a more central position in a network because they are connected to more nodes than others. Thus, psychometric $\mathrm{g}$ is a summative index of all links between the nodes rather than a specific process.

Mutualism is not entirely neutral about the interactions between processes in psychometric g. It assumes several constraints operating on these interactions. First, the very interaction between processes is a factor of growth and individual differences, because interactions exert a positive multiplier effect so that initial small differences multiply with time. 
A

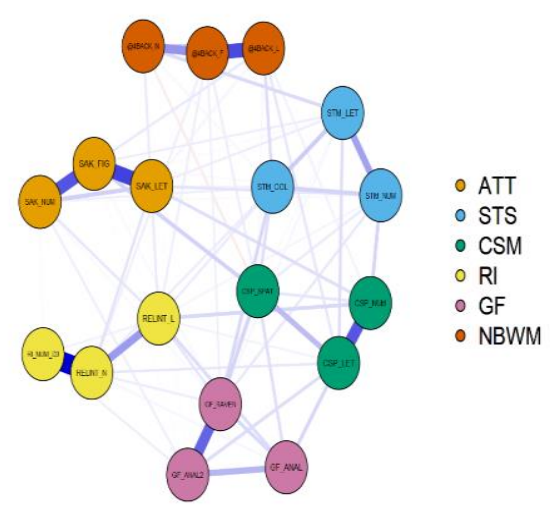

B

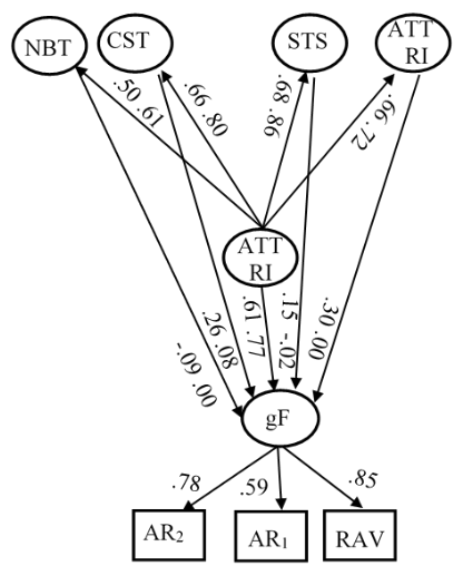

Figure 1. A network (A) and a structural equation model (B) of processes in $g$.

Note: The symbols ATT, STS, CST, RI, GF, NBWM, RAV, and AR, stand for attention control, short-term memory, complex span test, relational integration, fluid intelligence, n-back working memory, Raven, and analogical reasoning, respectively.

Second, small initial differences between processes due to genetic or environmental constraints may increase dramatically by the interactive dynamics in a Mathew-like effect (i.e., the rich get richer and the poor get poorer), causing both development and individual differences. Third, some processes may be more central in the network, such as working memory, thereby channeling the interactions and changes of the network in their direction (Savi et al., 2019; van der Maas et al., 2006, 2017). Recently, Process Overlap Theory (POT) emerged as a modern alternative to Thomson's (1916) sampling. POT claims that processing any task requires sampling multiple processes, recognizing that attention control or fluid intelligence are more central because they are used by most domain-specific processes, causing a bottleneck masking individual differences in different domains. Currently, POT adopted the foundational assumption 
of mutualism that $\mathrm{g}$ is an index of the interactions between the processes rather than a reflection of a specific psychological entity (Conway \& Kovacs, 2018; Kovacs \& Conway, 2016).

\section{Dynamic Mental Field Theory (DMFT)}

Mutualist and sampling theories face a serious problem. Technically, their assumption that the lack of $\mathrm{g}$ in networks is tantamount to nonexistence is unfounded. On the one hand, it is recently argued that it is mathematically possible to abstract hierarchical structure and thus $g$ from networks, under appropriate transformation of node loadings (Golino et al., 2020). On the other hand, interactions often reflect hidden entities to be identified. In the standard model of physics, entities, such as particles or planets, are assumed to give meaning to otherwise unexplainable interactions and they are often verified. Substantively, mutualist theories lack a meaning-making mechanism: speed, attention, or storage as such do not carry any meaning. Reifying interaction does not solve the problem because a mechanism is needed linking the environment with the organism, interpreting encounters, and generating behavior of value to the organism. Awareness and subjectivity must be part of intellectual functioning (Demetriou \& Kazi, 2001; Demetriou, Makris et al., 2018a). Empirical tests of this assumption are given below.

We suggested that this mechanism involves three inter-dependent processes: (i) Alignment, (ii) Abstraction, and (iii) Cognizance; the AACog mechanism (Demetriou et al., 2013, 2018a). Alignment is a "search, vary, and match" comparator process generating variations of stimuli or representations that may be linked according to current needs; it serves choice and decision optimization, given a current goal. Abstraction is a relational operator involving (i) identification of invariant characteristics across stimuli, (Burgoon et al., 2013; Demetriou, 2020), (ii) discrimination of characteristics to be excluded (Reed, 2016), and (iii) substitutivity, recognition that two or more elements may stand for the same referent (Gilead, 2019). AACog 
integrates recent research showing that relational integration is the minimal mechanism underlying gf. It comprises (1) mapping perceptually available stimuli with a single represented relation (Jastrzębski et al., 2020; (2) knowledge integration, i.e., mapping an inferred relation (e.g., larger than) with a corresponding relation stored in memory (e.g., horse $>\operatorname{dog}>$ mouse) (Hannon \& Daneman, 2014); (3) reduction of several variables into a single new cognitive representation (Dauvier et al., 2014; Oberauer et al. (2008).

Cognizance is the part of consciousness focusing on cognition. Cognizance comprises monitoring the alignment and abstraction processes, awareness of their objects and products, and reflection, i.e., revisiting mental processing and its objects and products to decide if satisfactory, given the moment's goal. Cognizance is meta-representational in that it recognizes that abstractions may be encoded into new representations. Cognizance is activated when abstractions and alignments are not satisfactory. Choosing between stimuli or actions turns the "mind's eye" to them thereby bringing them into the focus of awareness. Thus, cognizance allows feedback loops where cycles of abstraction and alignment may become the object of further abstraction and alignment which may be represented into new mental units. Enabling processes, such as attention or storage of information in sake of on-line meaning making, set constraints for alignment and abstraction. Widely investigated processes, such as Theory of Mind (Wellman, 2014), metacognition (Efklides, 2008), learning to learn (Demetriou, 2014), and growth mindset (Dweck, 2006) are expressions of cognizance and relate with g (e.g., Coyle et al., 2018; Demetriou \& Kazi, 2006; Demetriou et al., 2018b). We call the AACog mechanism "noetron", after nous, the Greek term for mind, as its basic noetic mechanism. Table 1 outlines the structure of noetron and compares with relevant processes in the brain and artificial intelligence to be discussed below. 
The study of mental processes and personality has been central in American psychology throughout $20^{\text {th }}$ century (Braat et al., 2020); however, awareness was never a legitimate construct of psychometric theories of intelligence. This might have been an epistemological tradeoff

Table 1. Noetron processes in the mind, the brain, and artificial intelligence.

\begin{tabular}{|c|c|c|c|}
\hline Process & Mind & Brain & $\mathrm{AI}$ \\
\hline Alignment & $\begin{array}{l}\text { Search, vary, and } \\
\text { match. }\end{array}$ & $\begin{array}{l}\text { Gain control mechanism } \\
\text { involving variation, search, } \\
\text { and integration of } \\
\text { perceptual information. }\end{array}$ & $\begin{array}{l}\text { Matching characteristics } \\
\text { according to an algorithm. } \\
\text { Traditional machine learning } \\
\text { models are reactive and can } \\
\text { do what they are } \\
\text { programmed to do (given a } \\
\text { dataset and a task to be } \\
\text { optimized, it performs the } \\
\text { task, but there is no self- } \\
\text { learning and adaptation). }\end{array}$ \\
\hline Abstraction & $\begin{array}{l}\text { Identification of } \\
\text { invariant } \\
\text { characteristics, } \\
\text { discrimination, } \\
\text { substitutivity. }\end{array}$ & $\begin{array}{l}\text { Integration networks, such } \\
\text { as temporo-parietal and } \\
\text { frontal areas; inferior } \\
\text { parietal and inferior frontal } \\
\text { cortices, von Economo } \\
\text { neuronal system, } \\
\text { coordination between } \\
\text { various oscillatory rhythms. }\end{array}$ & $\begin{array}{l}\text { Dropping of properties } \\
\text { unrelated to a task. } \\
\text { Interaction, selection, } \\
\text { evolution: capacity to learn } \\
\text { from the interaction with the } \\
\text { environment (and with the } \\
\text { feedback it receives), without } \\
\text { supervision (e.g, } \\
\text { reinforcement learning); } \\
\text { capacity to select the fittest } \\
\text { properties and to enable the } \\
\text { evolution of populations } \\
\text { (e.g., evolutionary generative } \\
\text { adversarial networks). }\end{array}$ \\
\hline Cognizance & $\begin{array}{l}\text { Awareness of mental } \\
\text { objects and processes, } \\
\text { and their products, } \\
\text { reflection, } \\
\text { metarepresentation. }\end{array}$ & $\begin{array}{l}\text { Anterior prefrontal cortex; } \\
\text { top-down attentional } \\
\text { amplification into a self- } \\
\text { sustained brain-scale state } \\
\text { of coherent activity that } \\
\text { involves many neurons } \\
\text { distributed throughout the } \\
\text { brain. }\end{array}$ & $\begin{array}{l}\text { Weak artificial } \\
\text { consciousness: being aware } \\
\text { of processes humans are } \\
\text { aware. } \\
\text { Strong artificial } \\
\text { consciousness: Becoming } \\
\text { aware of one's own } \\
\text { functioning thereby } \\
\text { becoming selective in the } \\
\text { fashion humans are. This } \\
\text { may not even be possible. }\end{array}$ \\
\hline
\end{tabular}


between the discipline of individual differences and behaviorism to secure its legitimacy as a scientific field, when behaviorism was prescribing that only observed behavior is a legitimate object of scientific study. In DMFT, cognizance is an integral part of noetron, co-determining its efficiency together with alignment and abstraction. It is recently suggested that the core function of consciousness is to enable subject-level experience which ascribes intrinsic values to mental states, allowing choices among them according to their value. In so doing it both unifies experience and allows options for action (Cleeremans \& Tallon-Baudry, 2021). In terms of DMFT, consciousness is the basic tool for creating options for better action in the future, given earlier experience. Any creature lacking consciousness would be unable to form an increasingly capable $\mathrm{g}$ as a tool for dealing with the unexpected by capitalizing on a balanced combination of past knowledge and experience with variations gauged against a future-oriented interpretation of the situation. Ideally, this combination must optimize choices by selecting the best fitting experience or concept to vary, based on how the future is conceived. For instance, many animals have high levels of intelligence ensuring perfect adaptation in their environments. However, if the environment changes at a scale they cannot be aware off and map to choose between corrective options, they would run the risk of extinction. Therefore, no theory of intelligence is meaningful without systematically integrating consciousnesses. Cognizance, as the aspect of consciousness focusing on cognition, affords special advantages, enabling the organism to take control of its own intelligence.

Noetron can be measured in several ways. Minimally, tasks would involve simple relational integration, such as singling out a perceptual relation implementing a represented 
relation (Jastrzębski et al., 2020). To contrast the operation of each AACog process with the operation of noetron as a unit, each process must be varied independently of the others to specify when meaning-making breaks down. For the present purposes, we focus on cognizance. For instance, variations at different times in the component stimuli of a relation to be identified would highlight when and how awareness becomes critical in identifying the relation. Presenting interference in integration may also function in this role. Alternatively, in a latent variable modeling approach, relational integration and cognizance tasks may be related to a latent noetron factor, whose relations with other factors, such as gf or WM, may be determined.

\section{In Search of the Ghost in the Mind}

\section{Critical Psychological Evidence}

The theories above yield contrasting predictions about the structure and development of the processes associated with g. Several models tested these predictions.

Is $g$ a process or an interaction? Mutualist theories predict that interactions between processes have special explanatory power per se, on top of the sum of individual processes involved. DMFT does not make any such assumption, because measures of noetron, if available, would be enough to account for measures of $\mathrm{g}$. To test this assumption, we used the data of Chuderski et al. (2014) summarized above. In one model we examined if the interactions between attention control, working memory, and relational integration processes predict performance on $\mathrm{gf}$ tasks on top of a general executive $\mathrm{g}$ factor $\left(\mathrm{g}_{\text {exec }}\right)$ underlying performance on these executive tasks. This mutualist prediction does not hold. The model involving no interaction fit better to the data than the interactive model, reflecting that $\mathrm{g}_{\text {exec }}$ accounted for a 
high amount of variance of each gf measure (all > .86), leaving no variance to be accounted for by any of the interactive effects.

Second, mutualist theories, especially POT, assume that executive processes are more central than other processes, causing the bottleneck effect. The DMFT sets noetron in the center of mental functioning. Thus, any measure of processes involved in noetron, such as relational integration, would be more powerful than executive processes in predicting $g$. These predictions require pitting attention control and relational integration against each other as mediators between gf and simpler constructs, such as working memory. Two models were tested in sake of this aim. In the first model, the attention control factor was taken as the mediator; thus, the three working memory factors, (short-term storage, complex span working memory, n-back working memory), and relational integration were regressed on attention control; gf was regressed on attention control and the residuals of all other factors. In the second model, relational integration was taken as the mediator; all three working memory factors and attention control were regressed on relational integration; gf was regressed on relational integration and the residuals of the other factors (Fig. 1B). Expectedly, gf was highly related to both attention control $(\beta=.61)$ and relational integration $(\beta=.77)$. However, in the first model, all factors but n-back working memory were significantly related with gf; in the second model, the relation of all factors with gf was close to 0 and non-significant. Therefore, relational integration is a stronger mediator between executive processes and gf than attention control.

What Develops in $g$ ? Noetron is the core of meaning-making activity running at different levels of understanding, from perception to advanced reasoning. At a basic cognitive level, noetron generates the fundamental building blocks of a Language of Thought (LoT) and, with development or learning, recombines them into increasingly complex inferential patterns. 
Recurrent relations in the environment are abstracted since infancy by Bayesian statistical inference mechanisms, such as conjunction (and, recurrent similarities), disjunction, (or, differences), etc., quantity (e.g., more than, larger than, etc.) (Piantodosi et al., 2016). When consolidated as distinct representations, they may be further used to integrate other representations, yielding deductive reasoning, mathematical reasoning, etc.

Changes with development in reasoning are reflected in changes in $\mathrm{g}$ but these relations vary with development, altering the profile of $g$ in successive developmental phases. This is suggested by studies examining changes in $\mathrm{g}$ as a function of development. Different theories specify these relations differently. Spearman's (1927) Law of Diminishing Returns, the psychometric interpretation of variation in the relations between $\mathrm{g}$ and specific processes, claims that specific processes differentiate from $g$ with development or increasing g. Developmental theory predicts increasing integration (Case, 1985; Piaget, 1970). DMFT posits that the very nature of $g$ varies across developmental phases, depending upon the developmental priorities of each phase. Developmental priorities vary with the functional state of noetron defining $\mathrm{g}$ (see Fig. 2). When a process is highly demanded for efficient noetron functioning, this process and $g$ integrate increasingly until the first becomes an integral part of the second. After a critical integration point in satisfying functional demands, the two may vary independently, because the formation of $\mathrm{g}$ shifts to other priorities showing dependence on other processes (Demetriou et al., 2017; Demetriou \& Spanoudis, 2018).

Several studies showed that attention control and representational awareness dominate from 4 to 7. Awareness of the perceptual origins of representations and attention control are important in this period because they allow to focus on representations of interest and organize actions accordingly. These processes are also important for mastering symbol systems such as 
language which occurs in this period. When mastered, these processes enable more complex cognitive tasks, such as planning, engagement in on-going verbal interactions, and exploring the behavior and interactions between other persons and objects. Inference is widely present in this phase. However, inference is still based on representations of episodes experienced: e.g., the child concludes seeing dad's car outside the house that "dad is in" because this is what usually happens (P and Q; P; therefore Q). However, accuracy of these inferences varies greatly because it is experience- rather than rule-based. As a result, reasoning in this phase it is not reflected in the profile of $g$.

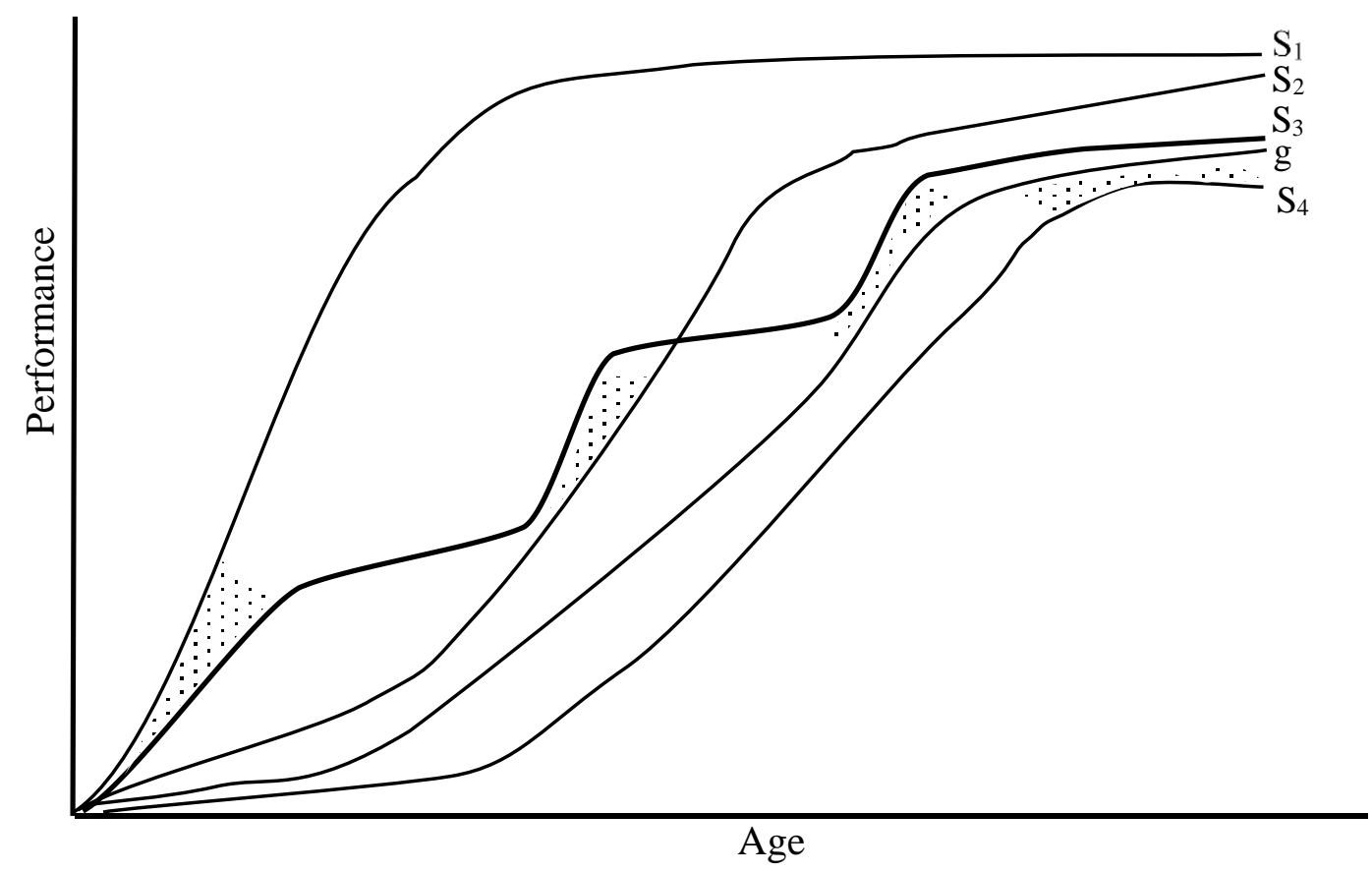

Figure 1. Shifting relations between $g$ and specific processes as a function of functional priorities of successive developmental phases.

Note: Shaded areas indicate regions of strengthened relations between $g$ and a specific process.

With attention control and representational awareness established, priorities change in primary school years, from 7 to 11 years. The relations between representations need to be 
worked out and accurately represented. Thus, cognitive priorities are redirected from linking representations with the environment and controlling activities accordingly to relations between representations themselves; connecting space-specific instances with extant concepts and interrelating concepts according to conceptual, procedural, or functional constraints become important. Children in this phase can revisit representations from alternative points of view grasping constant underlying relations, such as counting forward and backward according to a prespecified number, explicitly induce relations, and start to reason by rules rather than by experiential directives. For instance, they now explicitly represent conjunction relations as such, and they run them forward or backward, grasping modus tollens (i.e., P and Q; not Q; therefore not $\mathrm{P}$ ). Thus, in this phase, inductive inference, inferential awareness, and working memory dominate as markers of $\mathrm{g}$. Attention control and representational awareness disengage from the formation of $\mathrm{g}$, as $\mathrm{g}$ in this phase is formed by command of inferential rules.

The changes above are consolidated in adolescence. In this phase, deductive reasoning is fully established based on general principles prescribing when it is and when it is not possible to use an inferential rule to infer a state of reality based on this rule. For instance, they understand that an implication relation (if $\mathrm{P}$ then $\mathrm{Q}$ ) allows to infer $Q$ from $P$ and not $P$ from not $Q$, but it does not allow to infer $P$ from $Q$ or $Q$ from not $P$ because there may be an infinite number of other elements causing Q. Thus, deductive reasoning, awareness of logical principles, and precise cognitive self-representation dominate as markers of $\mathrm{g}$ from 12 to 17 years (Demetriou et al., 2013; Kazi et al., 2019; Makris et al., 2017). The processes dominating in the earlier periods differentiate. Obviously, awareness of the logical constraints imposed by principles directs searching for elements which are and which are not consistent with a mental model. These processes strengthen up to middle age. Notably, accurate cognitive self-evaluation and self- 
representation become powerful factors in the formation of $g$ among university educated persons in middle age. Later, in old age, advanced principle-based thought yields to rule-based thought, which again dominates as a marker of g over principle-based thought (Demetriou et al., 2017).

Relational complexity may be quantified and intellectual development may be described in terms of relational complexity (Douvier et al., 2014; Halford et al., 1998). However, the critical factor of relational integration is neither the sheer number of representations nor the number of representations defining a relation; the critical factor is the level of abstraction and cognizance at which relations are looked for. The mind's eye sees relations at the level at which these relations are sought; looking for low-level relations will uncover low-level relations, even if representational capacity is large. This approach explains a powerful empirical finding. There is a difference of about 2-3 units between short-term storage and working memory throughout the age span from 2 to 16 years, being 3 to 1 at 3 years and 6 to 3 at 16 years, respectively (Cowan, 2016; Gathercole, 2002). Obviously, children have the capacity to represent as many elements as required to grasp complex relations from preschool, but they do not because they are not oriented to searching, abstracting, and encoding these relations. Thus, it is notable that only in the phase from 7 to 11 working memory contributes to the formation of $\mathrm{g}$. It seems that increases in working memory in this period facilitates explicit rule induction dominating in this period; inversely, mastering rule use facilitates information management in working memory.

Where does change in $g$ come from? Training as a systematic experimental manipulation of relations between processes is a strong test of this model. Assuming that $\mathrm{g}$ is reformed in development according to the functional priorities of successive developmental phases implies that training any specific process would generalize to $g$ only if provided in the phase in which this process dominates in the formation of $\mathrm{g}$. Therefore, training executive processes, such as 
attention control and working memory, would generalize to reasoning only if provided in preschool or early primary school years. This training would not be effective in later years, when formation of $\mathrm{g}$ shifts to other processes. However, training relational integration and awareness of inferential processes in late childhood or adolescence would generalize to g because these processes are central in the formation of $\mathrm{g}$ in this period.

There is evidence in line with this pattern. Specifically, training attention control in 5years-old children enabled trained children to activate the executive attention network faster and more efficiently than untrained children several months after training and transferred to fluid intelligence (Rueda, Checa, \& Combita, 2012). Training working memory in 11-12 years old children improved the processes trained and transferred to school performance in English and mathematics (Holmes \& Gathercole, 2014), a proxy of $\mathrm{g}$ (Jensen, 1998). Training attention control and working memory in later years did not transfer to relational processes nor does it increase g or IQ (Melby-Lervåg, 2016; Sala \& Gobet, 2020; Shipstead et al., 2016). However, training relational integration (Klauer \& Phye, 2008; Papageorgiou et al., 2017; Shayer \& Adey, 2002; Vanikainen et al., 2020) and awareness and modeling deductive reasoning schemes (Christoforides et al., 2016) in late childhood and adolescence did improve reasoning and transferred to attention control and working memory.

In conclusion, cognitive training is not universally effective. It is effective if it specifically targets processes central in the formation of $\mathrm{g}$ in the developmental phase involved. Interestingly, the best predictors of school performance at different developmental phases are the processes defining $\mathrm{g}$ in each phase: attention control and representational awareness in preschool, inductive reasoning and working memory in primary school, and deductive reasoning 
and self-evaluation accuracy in adolescence (Demetriou Makris et al., 2020; Demetriou, Kazali et al., 2020; Demetriou, Kazi et al., 2019, 2020).

\section{An Agenda for 2050: Is There a Brain Noetron?}

Although mental structures, such as attention, memory, and reasoning need not correspond to brain structures on one-to-one basis (Pessoa et al., 2021), grounding the theory above in the brain would require demonstrating that brain functioning involves the (search-andvary), (align-and-abstract), and (integrate-and-cognize) processes involved in noetron. Evidence is supportive (see Table 1). Specifically, neural variability is an important aspect of brain functioning underlying successful behavior (Waschke et al., 2021). In fact, relational processing seems present at several levels of brain functioning. Perception itself is based on a gain control mechanism involving variation, search, and integration processes (Buzsaky, 2019). Saccadic eye movements and head movements or multimodal perception produce multiple object representations which are integrated in sake of recognition (Nanay, 2018). Gain control mechanisms coordinate representations across spaces abstracting object invariance (Ferguson $\&$ Cardin, 2020). Gain control is distributed over several brain regions, such as visual and acoustic cortices, and the hippocampal system, and it involves neural mechanisms capturing novelty: synaptic changes in hippocampal circuits activated when encountering new information relative to familiar information enable abstraction by signaling discrimination between known and new information (Gomex-Ocadic et al., 2021). Attention control is basically internalized gain control. 


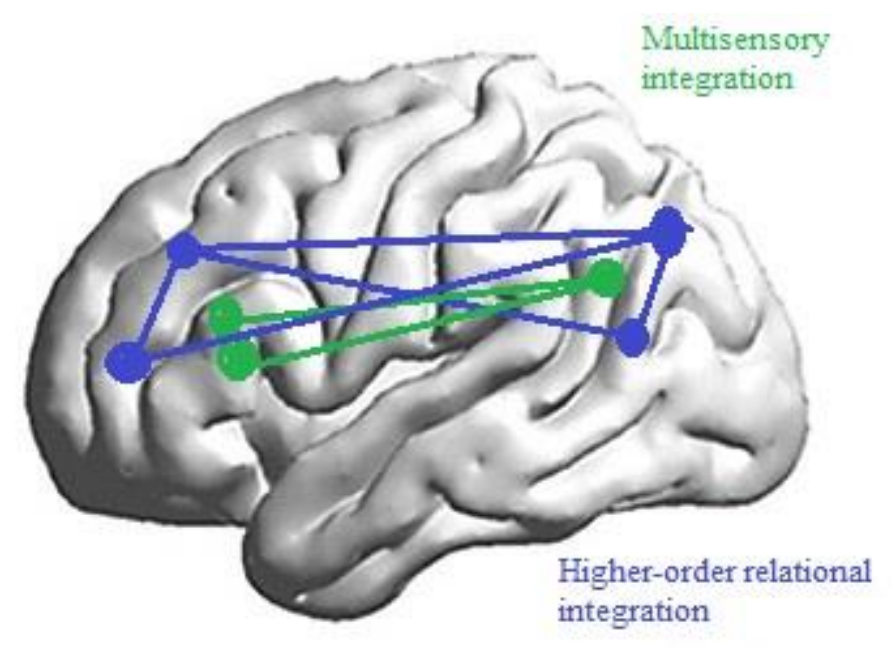

Figure 3. Networks of relational integration systems in the brain underlying noetron.

The partial overlap of relational processing at different mental levels is present in the partial overlap of the brain networks involved, which carry relations from perception to firstorder inferential connections to higher-order relations and syllogistically based relations. These networks are illustrated in Fig. 3. Specifically, integration of multisensory perception involves a modality-independent network in the inferior parietal (IPC/IPS) and inferior frontal cortices (IFG) which processes input from multiple senses. The IPS is implicated in allocating additional attentional resources when integrating multisensory stimuli and search for relatedness between stimuli from different senses. The IFC interlinks multisensory object representations with concepts in semantic memory, yielding meaning to stimuli (Porada et al., 2021). These regions partly overlap with a set of regions implicated in relational integration at a higher level. Comparative research suggests that the human brain, compared to other primate species, underwent evolutionary modifications which enriched the connectivity of the middle temporal lobe (Eichert et al., 2020) and adjacent parietal cortex (Mars et al., 2017) with other brain areas linked to relational thought, such as temporo-parietal and frontal areas (Goel et al., 2017). 
Posterior parietal cortex is implicated in the representation of first-order relations (e.g., A:B) and rostrolateral $\mathrm{pFC}$, implicated in integrating first-order relations to generate higher-order relations (e.g., A:B::C:D). These regions are also linked to dorsolateral $\mathrm{pFC}$ implicated in maintaining relations in working memory and ventrolateral $\mathrm{pFC}$, implicated in interference control (Holyoak \& Monti, 2020).

Explicit awareness of mental states always involves the prefrontal cortex. For instance, introspective ability correlated with grey matter volume and the white-matter microstructure in the anterior prefrontal cortex (Fleming et al., 2010). In fact, consciousness itself is a multilevel recurrent interactive state, emerging when a neural population related to a given mental state is mobilized by top-down attentional amplification into coherent activity throughout the brain. This makes this mental state explicitly cognized. Medial PFC and midline parietal cortex are always involved in this process and their impairment inflicts consciousness (Mashour et al., 2020).

Brain networks expand in cycles reminiscent of intellectual development (Spanoudis \& Demetriou, 2020). Bayesian rule extraction may start from as early as 3 months of age in anterior hippocampus, even before episodic memories are formed (Ellis et al., 2021), laying the ground for building blocks of LoT, when connections with the medial prefrontal cortex are established. Changes in the relations between $g$ and executive processes and reasoning at successive developmental cycles are related with the reformation of these networks. Thus, increases in intermodular connectivity by strengthening between-hub connections allow viewing each module from the point of view of other modules; decreases in intra-modular connectivity render the modules increasingly refined and task-specific. In this process some hubs become stronger and better connected to the modules involved, almost literally fleshing out rules and principles (Grayson et al., 2014; Oldham \& Fornito, 2019). That is, when activated as such, they allow 
activation to flow through the networks involved, delivering cases and implementations of the hub dominating. The more fluent the activation the better and faster the functioning; developmentally speaking, establishment of a network of this nature may correspond to a developmental cycle; psychometrically, differences in the efficiency of the activation of the network may underlie relevant individual differences (Demetriou \& Spanoudis, 2018; Spanoudis \& Demetriou, 2020; Haier, 2017).

The growth of these brain networks may relate with the coordination between various oscillatory rhythms, such as alpha, beta, gamma, and theta rhythms, reflecting activation of neural assemblies. For instance, high frequency rhythms, such as beta and gamma oscillations, stand for individual representations; these are enveloped into lower frequency rhythms, such as delta and theta rhythms, standing for integrated commonalities running through them (Buzsaki, 2006, 2019). Thus, the brain analogue of mental alignment of perceptions or representations may be oscillatory co-activations between the networks representing the mental entities involved. The functional brain equivalent of abstraction may be the lock of representation-specific rhythms in the all-inclusive rhythm (Leopold \& Park, 2020; Thut et al., 2012). Cognizance and metarepresentation may emerge when this new inclusive (e.g., theta) oscillation is somehow individuated and symbolized to become available later. It seems that temporal and rostrolateral prefrontal regions serve these needs (Dumontheil, 2014; Haier, 2017; Vatansever et., 2020).

Recently, a single class of neurons, the von Economo neurons (VEN), was named the "g-neuron" to suggest that it underlies relational integration between regions. It is assumed that VEN creates oscillatory coherence between distant regions, pairing a functionally invariant network of hub regions representing relations (Bruton, 2021). Therefore, differences in the population and functioning of VEN may relate with development and individual differences in 
g. Presumably, hubs in IPC, IPS, and IFG may be the structures serving AACog processes in noetron; VEN holds these structures functionally together. Different aspects of this network grow at different rates. VEN reaches adult numbers at about 4 years. Other aspects, such as myelinization and pruning, change until adulthood (Spanoudis \& Demetriou, 2020).

A critical test of the developmental priority model in the brain would be to show that the changes in the relations between $\mathrm{g}$ and specific processes outlined above occur at the level of the brain as well. For instance, reaching maturity by the VEN g-neuron at 4 years may relate with the fact that attention control is established from 4 to 7 years. Later in primary school, the relational network integrates with the reasoning network, allowing the emergence of inference as a marker of $\mathrm{g}$. In adolescence, the establishments of connections between the frontal and other relational integration regions allows explicit awareness to emerge as a strong marker of g. It is also worthwhile to examine if the interaction between the effectiveness of training experiments and developmental priorities summarized above alter the relations between the brain networks involved.

\section{An Agenda for 2100: Integrating Artificial, Biological, and Synthetic Intelligence}

The development of intelligent systems that can solve problems and act in the real world mimicking human beings is a long-standing goal of artificial intelligence. So far, however, no such system exists in modern approaches to artificial intelligence (AI) (see Table 1). In fact, learning in $\mathrm{AI}$ is based on complex machine learning algorithms (e.g., deep neural networks) which are fed with huge volumes of data so that they "discover" the regularities underlying a well-defined problem, such as face recognition or medical diagnosis (e.g., Do et al., 2018). Although successful, these machine learning programs are not really intelligent: their intelligence resides in the programmer writing their algorithms. Thus, they are limited in using a ready-made 
product to abstract regularities in a specific domain which without any generalization elsewhere. Even AI methods that involves abstraction, such as reinforcement learning techniques, evolutionary generative adversarial networks (Wang et al., 2019), and long short-term memory techniques, to name a few, are very limited in their applications. They involve, in general, the capacity to learn from the environment without supervision, to select the fittest properties and/or elements of the models, and to generate evolution to populations of intelligent agents. However, learning without supervision, selection of the fittest and evolution are very limited in these AI methods. They are limited by the constraints of what can be learned and how. They lack the necessary elements to learn by themselves, and to be aware of their own functioning, being selective, flexible, and adaptable (see Table 1).

For this to be possible, artificial intelligent systems must have a noetron-base $\mathrm{g}$ that can handle the unexpected. That is, they must be able to generate self-organized and adaptive cognitive possibilities undergoing human-like cognitive development (Lobov et al., 2020). Building Artificial General Intelligence (AGI) implies a system able to learn from complex, diverse, and open environments so that it can efficiently operate in them based on rules and representational models that systematically improve and can deal with the unexpected. Improvements must occur internally, without re-programming. This is reflected in reasoning routines able to generate new concepts and in "self-concepts" allowing choices, preferences, and evaluations (Adams et al., 2012; Goertzel, 2014). So far, no AGI creature exists. AI systems align, match, and abstract, but they not cognize. It was noted above that the core function of consciousness is to enable subject-level experience ascribing intrinsic values to events and memories, enabling a creature to choose among them and generate action according to them (Cleeremans \& Tallon-Baudry, 2021). A non-conscious creature lacks the basic tool for creating 
options for better future action, given past experience. No theory of AI can be successful in creating AGI in the fashion that no psychological theory of intelligence is meaningful without systematically integrating consciousnesses. So, let our robots become conscious and cognizant, in the fashion that our children become with growth.

It might be that neurally-based systems are more likely to develop in this direction rather than plain machine learning algorithms. One interesting candidate is the neurally controlled animat system (DeMarse et al., 2001). In this experiment, actual cortical tissue (neurons and glia) was abstracted from the rat and cultured since day 18 after conception. This culture was arranged in a multi-electrode array connected to a computer. The computer had a simulated environment with a digital rat receiving inputs from and sending outputs to the neuronal multielectrode array. The development of this chimeric system may give answers to several fundamental questions about how brains make minds (e.g., Nesbeth et al., 2016). Some of these questions are partially addressed in this paper: What are the fundamental neuronal principles underlying a cognitive system? How much are these principles determined by the microscopic cytoarchitecture of their primary neural substrates? What are the basic conditions in neural architecture that allows the development of g-like intelligence? When does a g-like capacity emerges in hybrid bio-synthetic intelligent systems? When do they become inter-relational and cognizant?

\section{The Future of Noetic Science: Capturing the Central Meaning-Making Unit}

Noetron was proposed as a central meaning-making unit of the human mind, implemented by the human brain, but still lacking in systems of artificial intelligence. Its basic function is to generate representations of the environment which have meaning for the organism: i.e., it gives meaning to objects by defining them in relation to each other, abstracting, 
representing, and metarepresenting their relations, and cognizing them, itself included, so that they can be reflected on, reintegrated at will, improved, and rendered available as understanding or action choices. Noetron expands by integrating increasingly efficient levels of control, going from episodic to representational to inferential to truth to epistemic control. Reasoning and problem solving in different domains are partly overlapping languages to be learned in each domain. In network terms, noetron may be seen as a small world architecture interacting with many other networks. Thus, "the capacity to flexibly transition between network states provides the foundation for individual differences in $\mathrm{g}$ - supporting the rapid exchange of information across networks and capturing individual differences in cognitive processing at a global level." (Barbey, 2018). However, this is not any small world architecture network. Self-knowledge is built in it by construction, participating in its transitions, even when wrong. Going bottom up, evolution took millennia to deliver it in the human brain. Artificial intelligence, designing applications capitalizing on some of its most advanced products, failed to simulate it, so far.

Representational precision is an important but underestimated issue in understanding how transitions occur. That is, how the representation of the fundamental information unit in different domains at different developmental or learning phases is effected. For instance, in language, word sounds in infancy, word meaning in preschool, integration of visual and acoustic patterns in reading in primary school, and abstract semantic meaning in adolescence. In mathematics, subitatization sets, magnitudes, cardinal and ordinal number, algebraic number. Often, these representations are not precise enough; thus, their relations are either abstracted wrongly or not abstracted at all, handicapping emergence of new relational structures. It is important to specify representational precision both at the psychological and the brain level. For instance, at the psychological level, behavioral manifestations of relational integration and corresponding 
cognizance constructs must be compared to specify how they match. At the level of the brain, the focus and efficiency of brain activation of the cell assemblies involved and oscillatory activity carrying the networking of these assemblies in individuals clearly differing in representational precision must be mapped and compared. Also, specifying relations between changes in representational precision and in focus and efficiency of brain activation in longitudinal growth or change due to learning would bring psychological and modeling of intelligence research closer.

For years, development was thought to go through a necessary developmental sequence and individual differences in how far one may go along this sequence were thought as stable. Recent developments do not justify these positions. The Flynn (2009) effect suggests that g does change. Recent research suggests that this trend continues (Must \& Must, 2020), although there is evidence that change in some aspects of g may halt (Flynn \& Shayer, 2018). Can positive trends be expanded technically? Artificial crystallized intelligence has already improved hugely: Google Scholar and Wikipedia are vast pools of knowledge to which everyone may turn. Shall we have similar tools for $\mathrm{g}$ and gf to which one may turn for problem solving? Yes, if AGI as defined above would become a reality.

For this to occur, questions such as the following would have to be answered: What is required to simulate a 3-year-old child? For instance, is training attention control and perceptual awareness necessary to make this AI system operate under representational self-guidance? What must be added to make this system develop into an 8-year-old "artificial" child? Is training inductive reasoning, inferential awareness, and management of working memory necessary for this transition? Shall we get a 17-year-old "artificial" adolescent if logical awareness and skill in constructing mental models of logical relations are added? Noeborgs will be born when precise 
answers to these questions will be given. When Noeborgs will start going around, then we will have a big problem to solve: how do we deal with our own kids when they will start asking for Noeborgs as their toys?

\section{References}

Adams, S. S., Arel, I., Bach, J., et al., (2012). Mapping the landscape of human-level artificial general intelligence. AI Magazine, 33, 25-41.

Baddeley, A. D. (2012). Working memory: Theories, models, and controversies. Annual Review of Psychology, 63, 1-29.

Barbey, A. K. (2018). Network neuroscience theory of human intelligence. Trends in Cognitive Sciences, 22, https://doi.org/10.1016/j.tics.2017.10.001

Braat et al. (2020). The rise and fall of behaviorism: The narrative and the numbers. History of Psychology, 23, 252-280.

Butterworth, B. (2010). Foundational numerical capacities and the origins of dyscalculia. Trends in Cognitive Sciences, 14, 534-541. doi:10.1016/j.tics.2010.09.007

Carroll, J. B. (1993). Human cognitive abilities: A survey of factor-analytic studies; New York: Cambridge University Press.

Case, R. (1985). Intellectual development. Birth to adulthood. New York: Academic Press.

Case, R., Demetriou, A., Platsidou, M., \& Kazi, S. (2001). Integrating concepts and tests of intelligence from the differential and the developmental traditions, Intelligence, 29, 307336.

Cleeremans, A., \& Tallon-Baudry, C. (2021, May 18). The function of consciousness is to generate experience. PysArXiv, https://doi.org/10.31234/osf.io/jfpw2

Conway, T. R., Elpers, K. E., Gonzalz, M. C. Freeman, J., Beggio, J. A. (2018). General 
Intelligence (g), ACT Scores, and Theory of Mind: (ACT)g predicts limited variance among Theory of Mind tests. Intelligence, 71, 85-91. https://doi.org/10.1016/j.intell.2018.10.006

Bruton, O. J. (2021). Is there a "g-neuron"? Establishing a systematic link between general intelligence $(\mathrm{g})$ and the von Economo neuron. Intelligence, 86, 101540. https://doi.org/10.1016/j.intell.2021.101540

Burgoon, E. M., Henderson, M. D. \& Markman, A. B. (2013) There are many ways to see the forest for the trees: A tour guide for abstraction. Perspectives on Psychological Science 8, $501-520$.

Butterworth, B. (2010). Foundational numerical capacities and the origins of dyscalculia. Trends in Cognitive Sciences, 14, 534-541. doi:10.1016/j.tics.2010.09.007

Buzsaki, G. (2006). Rhythms of the brain. New York, NY: Oxford University Press.

Buzsaki, G. (2019). The brain from inside out. New York, NY: Oxford University Press

Carroll, J. B. (1993). Human cognitive abilities: A survey of factor-analytic studies; New York: Cambridge University Press.

Case, R. (1985). Intellectual development. Birth to adulthood. New York: Academic Press.

Case, R., Demetriou, A., Platsidou, M., \& Kazi, S. (2001). Integrating concepts and tests of intelligence from the differential and the developmental traditions, Intelligence, 29, 307336.

Christoforides, M., Spanoudis, G., Demetriou, A. (2016). Coping with logical fallacies: A developmental training program for learning to reason. Child Development, 87, 18561876.

Chuderski,A.(2014). The relational integration task explains fluid reasoning above and beyond 
other working memory tasks. Memory \& Cognition, 42, 448-463.

https://doi.org/10.3758/s13421-013-0366-x.

Cleeremans, A., \& Tallon-Baudry, C. (2021, May 18). The function of consciousness is to generate experience. PsyArXiv, https://doi.org/10.31234/osf.io/jfpw2.

Cliffordson, C., \& Gustafsson, J.-R. (2008). Effects of age and schooling on intellectual performance: Estimates obtained from analysis of continuous variation in age and length of schooling. Intelligence, 36, 143-152. http://dx.doi.org/10.1016/j.intell.2007.03.006

Conway, A. R. A., Cowan, N., Bunting, M. F., Theriault, D., J., \& Minkoff, S. R. B. (2002). A latent variable analysis of working memory capacity, short-term memory capacity, processing speed, and general fluid intelligence. Intelligence, 30, $163-183$.

Conway, A. R. A. \& Kovacs, K. (2018). The nature of the general factor of intelligence. In R. J. Sternberg, (Ed.), The general factor of intelligence (pp. 49-63). Oxford: Oxford University Press.

Cowan, N. (2016). Working memory maturation: Can we get at the essence of cognitive growth? Perspectives on Psychological Science, 11, 239-264. DOI:

$10.1177 / 1745691615621279$

Coyle et al., (2018). General intelligence (g), ACT scores, and Theory of Mind: (ACT)g predicts limited variance among Theory of mind tests. Intelligence, 71, 85-91. https://www.sciencedirect.com/science/journal/01602896

Dauvier, B., Bailleux, \& Perret, P. (2014). The development of relational integration during childhood. Developmental Psychology, 50, 1687-1697. DOI: 10.1037/a0036655

Dehaene, S. (2010). Reading in the brain: The new science of how we read. New York: Penguin.

DeMarse, T., B., Wagenar, D. A., Blau, A. W., \& Potter, S. M. (2001). The neurally controlled 
animat: Biological brains acting with simulated bodies. Autonomous Robots 11, 305-310.

Demetriou, A. (2014). Learning to learn, know, and reason. In R. Deakin-Crick \& C. Stringer, C. (Eds.), Learning to learn (43-68). London: Routledge.

Demetriou, A. (2020). Abstracting abstraction in development and cognitive ability. Behavioral and Brain Sciences, 43, DOI:10.1017/S0140525X19002930

Demetriou, A., Greiff, S., Makris, N., Spanoudis, G., Panaoura, R., \& Kazi, S. Bridging. (2020). educational priorities with developmental priorities: Towards a developmental theory of instruction. PsyArXiv, https://doi.org/10.31234/osf.io/kjmu3

Demetriou, A., \& Kazi, S. (2001). Unity and modularity in the mind and the self: Studies on the relationships between self-awareness, personality, and intellectual development from childhood to adolescence. London: Routledge.

Demetriou, A., \& Kazi, S. (2006). Self-awareness in g (with processing efficiency and reasoning). Intelligence, 34, 297-317.

Demetriou, A., Kazali, E., \& Kazi, S., \& Spanoudis, G. (2020). Cognition and cognizance in preschool predict school achievement in primary School. Cognitive Development, https://doi.org/10.1016/j.cogdev.2020.100872

Demetriou, A., Kazi, S., Spanoudis, G., \& Makris, N. (2019). Predicting school performance from cognitive ability, self-representation, and personality from primary school to senior high school. Intelligence, 76, https://doi.org/10.1016/j.intell.2019.101381

Demetriou, A., Kazi, S., Spanoudis, G., Makris, N. (2020). Cognitive ability, cognitive selfawareness, and school performance: From childhood to adolescence. Intelligence, 79, https://doi.org/10.1016/j.intell.2020.101432.

Demetriou, A., Makris, N., Kazi, S., Spanoudis, G., \& Shayer, M. (2018a). The developmental 
trinity of mind: Cognizance, executive control, and reasoning. WIREs Cognitive Science, 2018;e1461. https://doi.org/10.1002/wcs.1461

Demetriou, A., Makris, N., Kazi, S., Spanoudis, G., Shayer, M., \& Kazali, E. (2018b). Mapping the dimensions of general intelligence: An integrated differential-developmental theory. Human Development, 61, 4-42. http://dx.doi.org/10.1159\%2F000484450.

Demetriou, A., Makris, N., Tachmatzidis, D., Kazi, S., \& Spanoudis, G. (2019). Decomposing the influence of mental processes on academic performance. Intelligence, 77 , https://doi.org/10.1016/j.intell.2019.101404

Demetriou, A., \& Spanoudis, G. (2018). Growing minds: A developmental theory of intelligence, brain and education. London: Routledge.

Demetriou, A., Spanoudis, G., Shayer, M., Mouyi, A., Kazi, S., \& Platsidou, M. (2013). Cycles in speed-working memory-G relations: Towards a developmental-differential theory of the mind. Intelligence, 41, 34-50, doi: 10.1016/j.intell.2012.10.010.

Demetriou, A., Spanoudis, G., Kazi, S., Mouyi, A., Žebec., M. S., Kazali, E., Golino, H. F., Bakracevic, K., \& Shayer (2017). Developmental differentiation and binding of mental processes with re-morphing g through the life-span. Journal of Intelligence, 5, 23; doi:10.3390/jintelligence5020023.

Diamond, A. (2013). Executive functions. Annual Review of Psychology, 64,135-168.

Do, T. D., Duong, M. T., Dang, Q. V., \& Le, M. H. (2018, November). Real-time self-driving car navigation using deep neural network. In 2018 4th International Conference on Green Technology and Sustainable Development (GTSD) (pp. 7-12). IEEE.

Dumontheil, I. (2014). Development of abstract thinking during childhood and adolescence: The role of rostrolateral prefrontal cortex. Developmental Cognitive Neuroscience, 10, 57-76. 
Dweck, C. S. (2006). Mindset: The new psychology of success. New York, NY: Ballantine Books.

Efklides, A. (2008). Metacognition: Defining its facets and levels of functioning in relation to self-regulation and co-regulation. European Psychologist, 13, 277-287.

Eichert, N., Robinson, E., Bryant, K. L., et al., (2020). Cross-species cortical alignment identifies different types of anatomical reorganization in the primate temporal lobe. eLife 2020;9:e53232. https://doi.org/10.7554/eLife.53232,

Ellis, C. T., Skalaban, L. J., Yates, T. S., Bejjanki, V. R., Córdova, N. I., Turk-Browne, N. B. (2021). Evidence of hippocampal learning in human infants. Current Biology, in press, https://doi.org/10.1016/j.cub.2021.04.072.

Epskamp S., Maris G., Waldorp L. J. \& Borsboom D. (2018) Network psychometrics. In: Handbook of psychometrics, ed. P. Irwing, D. Hughes \& T. Booth, pp. 953-87. Wiley.

Ferguson, K. A., \& Cardin, J. A. (2020). Mechanisms underlying gain modulation in the cortex. Nature Reviews Neuroscience, 21, 80-92.

Fleming, S. M., Weil, R. S., Nagy, Z., Dolan, R. J., \& Rees, G. (2010). Relating introspective accuracy to individual differences in brain structure. Science, 329, 1541-1543.

Flynn, J. R. (2009). What is intelligence: Beyond the Flynn effect. Cambridge: Cambridge University Press

Flynn, J. R., \& Shayer, M. (2018). IQ decline and Piaget: Does the rot start at the top? Intelligence, 66, 112-121. https://doi.org/10.1016/j.intell.2017.11.010

Gathercole, S. A. (2002). Memory development during the childhood years. In A. Baddelley, M D. Kopelman, \& B, A. Wilson, (Eds.), The handbook of memory disorders (pp. 475-499). London: Wiley \& Sons. 
Gilead, M., Trope, Y., Liberman, N. (2020). Above and beyond the concrete: The diverse representational substrates of the predictive brain. Behavioral and Brain Sciences, 43, e121: 1-74. doi:10.1017/S0140525X19002000

Goertzel, B. (2014). Artificial general intelligence: Concept, state of the art, and future prospects. Journal of Artificial General Intelligence, 5, 1-46. DOI: 10.2478/jagi-20140001.

Goel, V., Navarrete, G., Noveck, I. A., \& Prado, J. (2017). The reasoning brain: the interplay between cognitive neuroscience and theories of reasoning. Frontiers in human neuroscience, 10, 673.

Golino, H., Thiyagarajan, J. A., Sadana, R., Teles, M., Christensen, A. P., \& Boker, S. M. (2020, October 19). Investigating the broad domains of intrinsic capacity, functional ability and environment: An exploratory graph analysis approach for improving analytical methodologies for measuring healthy aging. https://doi.org/10.31234/osf.io/hj5mc

Gomex-Ocadic et al. (2021). A synaptic novelty signal in the dentate gyrus supports switching hippocampal attractor networks from generalization to discrimination. bioR $\chi \mathrm{iv}$, doi: https://doi.org/10.1101/2021.02.24.432612

Gottfredson, L. S. (2002) Where and Why g Matters: Not a Mystery, Human Performance, 15, 25-46, DOI: 10.1080/08959285.2002.9668082

Grayson, D. S., Ray, S., Carpenter, S., Iyer, S., Dias, T. G. C., Stevens, C., ... \& Fair, D. A. (2014). Structural and functional rich club organization of the brain in children and adults. PloS one, 9(2), e88297.

Gustafsson, J. -E. (1984). A unifying model for the structure of intellectual abilities. Intelligence, 8, 179-203. 
Haier, R. J. (2017). The neuroscience of intelligence. Cambridge: Cambridge University Press.

Halford, G. S., Wilson, W. H., \& Phillips, S. (1998). Processing capacity defined by relational complexity: Implications for comparative, developmental, and cognitive psychology. Behavioral and Brain Sciences, 21, 803-864.

Hannon, B., Daneman, M. (2014). Revisiting the construct of "relational integration" and its role in accounting for general intelligence: The importance of knowledge integration. Intelligence, 47, 175-187. http://dx.doi.org/10.1016/j.intell.2014.09.010

Holmes, J., \& Gathercole, S. E., (2014) Taking working memory training from the laboratory into schools. Educational Psychology, 34, 440-450, 10.1080/01443410.2013.797338

Holyoak, K., \& Monti, M. (2020). Relational integration in the human brain: A review and synthesis. Journal of Cognitive Neuroscience, 33, 341-356, https://doi.org/10.1162/jocn_a_01619

Hughes, C., \& Leekham, S. (2004). What are the links between theory of mind and social relations? Review, reflections and new directions for studies of typical and atypical development. Social Behavior, 13, 590-619

Kail, R. (2000). Speed of information processing: Developmental change and links to intelligence. Journal of School Psychology, 38, 51-61.

Kazi, S., Kazali, E., Makris, N., Spanoudis, G., \& Demetriou, A., (2019). Cognizance in cognitive development: A longitudinal study. Cognitive Development, 52, https://doi.org/10.1016/j.cogdev.2019.100805

Kovacs, K., \& Conway, A. R. A. (2016). Process overlap theory: A unified account of the general factor of intelligence. Psychological Inquiry, 27, 151-177.

Gottfredson, L. S. (2002) Where and Why g Matters:Not a Mystery, Human Performance, 15, 
25-46, DOI: 10.1080/08959285.2002.9668082.

Jastrzębski, J., Ociepka, M., \& Chuderki, A. (2020). Fluid reasoning is equivalent to relation processing. Intelligence, 82, https://doi.org/10.1016/j.intell.2020.101489

Jensen, A. R (1998). The g factor: The science of mental ability. Westport, CT: Praeger.

Jensen, A. R. (2006). Clocking the mind: Mental chronometry and individual differences. Amsterdam: Elsevier.

Kail, R. (2000). Speed of information processing: Developmental change and links to intelligence. Journal of School Psychology, 38, 51-61.

Khooshabeh, P., Hegarty, M., \& Shipley, T. F. (2013). Individual differences in mental rotation: Piecemeal versus holistic processing. Experimental Psychology, 60, 164-171.

Klauer, K.J. \& Phye, G.D. (2008). Inductive Reasoning: A Training Approach. Review of Educational Research, 78(1), 85-123.

Kovacs, K., \& Conway, A. R. A. (2019). What is IQ? Life beyond "General Intelligence". Current Directions in Psychological Science, 28, 189-194, https://doi.org/10.1177/09637214198272

Leopold, D. A., \& Park, S. H. (2020). Studying the visual brain in its natural rhythm. Neuroimage, 216, 116790.

Lobov, S. A., Chernyshov, A. V., Krilova, N. P., Shamshin, M. O., and Kazantsev, V. B. (2020). Competitive learning in a spiking neural network: towards an intelligent pattern classifier. Sensors 20:E500. doi: 10.3390/s20020500

Makris, N., Tahmatzidis, D., Demetriou, A., \& Spanoudis, G. (2017). Mapping the evolving core of intelligence: Relations between executive control, reasoning, language, and awareness. Intelligence, 62, 12-30. 
Mars, R. B., Passingham, R. E., Neubert, F. X., Verhagen, L, \& Sallet J. (2017). Evolutionary specializations of the human association cortex. In: Kaas H. J (Ed). Evolution of Nervous systems. Oxford: Elsevier. p. 1-37. DOI: https://doi.org/10.1016/B978-0-12-804042$3.00118-4$

Mashour, G. A., Roelfsema, P., Changeux, J.-P., \& Dehaene, S. (2020). Conscious processing and the global neuronal workspace hypothesis. Neuron, 105, doi.org/10.1016/j.neuron.2020.01.026.

Melby-Lervåg, M., Redick, T. S., Hulme, C. (2016). Working memory training does not improve performance on measures of intelligence or other measures of "far transfer": Evidence from a meta-analytic review. Perspectives on Psychological Science, 11, 512-34.

Moshman, D. (2011). Adolescent rationality and development: Cognition, morality, and identity. (3rd ed.). New York, NY: Psychology Press.

Must, O., \& Must, A. (2020). No decline in the principle-based thought of 9-year-old schoolchildren between 1991 and 2001. Intelligence, 80, 101455. https://doi.org/10.1016/j.intell.2020.101455.

Nanay, B. (2018). Multimodal mental imagery. Cortex, 105, 125-134.

Nesbeth, D. N., Zaikin, A., Saka, Y., Romano, M. C., Giuraniuc, C. V., Kanakov, O., \& Laptyeva, T. (2016). Synthetic biology routes to bio-artificial intelligence. Essays in Biochemistry, 60, 381-391.

Oldham, S., \& Fornito, A. (2019). The development of brain network hubs. Developmental cognitive neuroscience, 36, 100607.

Oberauer, K., Süß, H. -M., Wilhelm, \& Wittman, W.W. (2008). Which working memory functions predict intelligence? Intelligence, 36, 641-652. 
Papageorgiou, E., Christou, C., Spanoudis, G., \& Demetriou, A. (2016). Augmenting intelligence: Developmental limits to learning-based cognitive change. Intelligence, $56,16-27$.

Pascual-Leone, J., \& Johnson, J. (2021). The working mind. Cambridge MA, MIT Press.

Pessoa, L., Medina, L., \& Desfilis, E. (2021). Mental categories and the vertebrate brain: the neural basis of behavior. https://doi.org/10.31219/osf.io/8cmhg

Piaget, J. (1970). Piaget's theory. In P. H. Mussen (Ed.), Carmichael's handbook of child development (pp. 703-732). New York, NY: Wiley.

Piaget, J. (2001). Studies in reflecting abstraction. London: Psychology Press.

Piantadosi, S. T., Tenenbaum, J. \& Goodman, N. (2016). The logical primitives of thought: Empirical foundations for compositional cognitive models. Psychological Review, 123, 392-424. http://dx.doi.org/10.1037/a0039980.

Porada, D., Regenbogen, C., Freiherr, J., Seubert, J., \& Lundstrom, J. N. (2021). Trimodal processing of complex stimuli in inferior parietal cortex is modality-independent. Cortex, 139, 198-210. https://doi.org/10.1016/j.cortex.2021.03.008

Protzko, J. (2015). The environment in raising early intelligence: A meta-analysis of the fadeout effect. Intelligence, 53, 202-210.

Reed, S. K. (2016) A taxonomic analysis of abstraction. Perspectives on Psychological Science 11, 817-37.

Revelle, W., \& Wilt, J. (2013). The general factor of personality: A general critique. Journal of Research in Personality, 47, 493-504.

Rindermann, H., \& Ackerman, A. L. (2020). Piagetian tasks and psychometric intelligence: Different or similar constructs? Psychological Reports, 
DOI: $10.1177 / 0033294120965876$.

Rueda, R. M., Checa, P., \& Combita, L. M. (2012). Enhanced efficiency of the executive attention network after training in preschool children: Immediate changes and effects after two months. Developmental Cognitive Neuroscience, 25, S192-S204. doi:10.1016/j.den.2011.09.004

Salthouse, T. A. (2016). Theoretical perspectives on cognitive aging. London. Routledge.

Sala, G., \& Gobet, F. (2020). Working memory training in typically developing children: A multilevel meta-analysis. Psychonomic Bulletin \& Review, 27, 423-434. https://doi.org/10.3758/s13423-019-01681-y

Savi, A. O., Marsman, M., van der Maas, H. L. J., \& Maris, G. K. J. (2019). The wiring of intelligence. Perspectives on Psychological Science, 14 (6), 1034-1061. https://doi.org/10.1177/1745691619866447.

Shayer, M. \& Adey, P.S, (2002) (eds.). Learning Intelligence: Cognitive Acceleration across the curriculum from 5 to 15 years. Milton Keynes: Open University Press.

Shipstead, Z., Harrison, T. L., \& Engle, R. W. (2016). Working memory capacity and fluid intelligence: Maintenance and disengagement. Perspectives on Psychological Science, 11, 771-799. DOI: $10.1177 / 1745691616650647$.

Spanoudis, G., \& Demetriou, A. (2020). Mapping mind-brain development: Towards a comprehensive theory. Journal of Intelligence, 8, 19, http://dx.doi.org/10.3390/jintelligence8020019.

Spearman, C. (1904). "General intelligence," Objectively determined and measured. The American Journal of Psychology, 15, 201-292.

Spearman, C. (1927). The abilities of man: Their nature and measurement. London: MacMillan. 
Thomson, G. H. (1916). A hierarchy without a general fac-tor. British Journal of Psychology, 1904-1920, 8, 271-281.http://dx.doi.org/10.1111/j.2044-8295.1916.tb00133.x

Thut, G., \& Carlo, M. (2009). New insights into rhythmic brain activity from TMS-EEG studies. Trends in Cognitive Sciences, 134, 182-89.

van Bork, R., Epskamp, S., Rhemtulla, M., Borsboom, D., \& van der Maas, H. L. (2017). What is the p-factor of psychopathology? Some risks of general factor modeling. Theory \& Psychology, 27, 759 -773. http://dx .doi.org/10.1177/0959354317737185

van der Maas, H. L. J., Dolan, C. V., Grasman, R. P. P. P., Wicherts, J. M., Huizenga, H. M., \& Raijmakers, M. E. J. (2006). A dynamical model of general intelligence: The positive manifold of intelligence by mutualism. Psychological Review, 113 (4), 842-861. https://doi.org/10.1037/0033-295x.113.4.842

van der Maas, H. L. J., Kan, K.-J., Marsman, M., \& Stevenson, C. E. (2017). Network models for cognitive development and intelligence. Journal of Intelligence, 5, 16. https://doi.org/10.3390/jintelligence5020016.

Vainikainen, M.-P., \& Hautamäki, J. (2020): Three studies on Learning to Learn in Finland: Anti-Flynn effects 2001-2017, Scandinavian Journal of Educational Research, DOI: 10.1080/00313831.2020.1833240.

Vatansever, D., Schröter, M., Adapa, R. M., Bullmore, E. T., Menon, D. K., \& Stamatakis, E. A. (2020). Reorganisation of brain hubs across altered states of consciousness. Scientific reports, 10, 1-11.

Wellman, H.M. (2014). Making minds: How theory of mind develops. Oxford University Press: Oxford, UK.

Wang, C., Xu, C., Yao, X., \& Tao, D. (2019). Evolutionary generative adversarial 
networks. IEEE Transactions on Evolutionary Computation, 23(6), 921-934. 